\title{
A Bayesian Approach to Bandwidth Selection for Multivariate Kernel Density Estimation
}

\author{
Xibin Zhang Maxwell L. King* Rob J. Hyndman \\ Department of Econometrics and Business Statistics \\ Monash University, Clayton, Victoria 3800, Australia
}

November 2004

Revised: May 2005

\begin{abstract}
Kernel density estimation for multivariate data is an important technique that has a wide range of applications. However, it has received significantly less attention than its univariate counterpart. The lower level of interest in multivariate kernel density estimation is mainly due to the increased difficulty in deriving an optimal data-driven bandwidth as the dimension of the data increases. We provide Markov chain Monte Carlo (MCMC) algorithms for estimating optimal bandwidth matrices for multivariate kernel density estimation. Our approach is based on treating the elements of the bandwidth matrix as parameters whose posterior density can be obtained through the likelihood cross-validation criterion. Numerical studies for bivariate data show that the MCMC algorithm generally performs better than the plug-in algorithm under the Kullback-Leibler information criterion, and is as good as the plug-in algorithm under the mean integrated squared error (MISE) criterion. Numerical studies for five dimensional data show that our algorithm is superior to the normal reference rule. Our MCMC algorithm is the first datadriven bandwidth selector for multivariate kernel density estimation that is applicable to data of any dimension.
\end{abstract}

Keywords: Cross-validation; Kullback-Leibler information; Mean integrated squared errors; Sampling algorithms; Monte Carlo kernel likelihood

\footnotetext{
*Corresponding author. Tel.: +61-3-99052449; fax: +61-3-99058039.

E-mail address: max.king@buseco.monash.edu.au (M.L. King).
} 


\section{Introduction}

Multivariate kernel density estimation is an important technique in multivariate data analysis and has a wide range of applications (see, for example, Scott, 1992; Aït-Sahalia, 1996; Donald, 1997; Stanton, 1997; Aït-Sahalia and Lo, 1998; de Valpine, 2004). However, its widespread usefulness has been limited by the difficulty in computing an optimal datadriven bandwidth. We remedy this deficiency in this paper.

Let $\boldsymbol{X}=\left(X_{1}, X_{2}, \ldots, X_{d}\right)^{\prime}$ denote a $d$-dimensional random vector with density $f(\boldsymbol{x})$ defined on $\boldsymbol{R}^{d}$, and let $\left\{\boldsymbol{x}_{1}, \boldsymbol{x}_{2}, \ldots, \boldsymbol{x}_{n}\right\}$ be an independent random sample drawn from $f(\boldsymbol{x})$. The general form of the kernel estimator of $f(\boldsymbol{x})$ is (Wand and Jones, 1995):

$$
\hat{f}_{H}(\boldsymbol{x})=\frac{1}{n} \sum_{i=1}^{n} K_{H}\left(\boldsymbol{x}-\boldsymbol{x}_{i}\right)
$$

where $K_{H}(\boldsymbol{x})=|H|^{-1 / 2} K\left(H^{-1 / 2} \boldsymbol{x}\right), \quad K(\cdot)$ is a multivariate kernel function, and $H$ is a symmetric positive definite $d \times d$ matrix known as the bandwidth matrix.

The bandwidth matrix can be restricted to a class of positive definite diagonal matrices, and then the corresponding kernel function is known as a product kernel. However, there is much to be gained by choosing a full bandwidth matrix, where the corresponding kernel smoothing is equivalent to pre-rotating the data by an optimal amount and then using a diagonal bandwidth matrix (Wand and Jones, 1993). It has been widely recognized that the performance of a kernel density estimator is primarily determined by the choice of bandwidth, and only in a minor way by the choice of kernel function (see, for example, Izenman, 1991; Scott, 1992; Simonoff, 1996).

A large body of literature exists on bandwidth selection for univariate kernel density 
estimation (see, for example, Marron 1987; Jones, Marron and Sheather 1996 for surveys). However, the literature on bandwidth selection for multivariate data is very limited. To choose an optimal bandwidth matrix, a criterion must be used; one such criterion is the integrated squared error (ISE) given by

$$
\operatorname{ISE}(H)=\int_{R^{d}}\left[\hat{f}_{H}(\boldsymbol{x})-f(\boldsymbol{x})\right]^{2} d \boldsymbol{x},
$$

where $f(\boldsymbol{x})$ denotes the target density. The least-squares cross-validation method aims to derive a bandwidth that minimizes ISE (see, for example, Härdle, 1991; Sain, Baggerly and Scott, 1994). However, the difficulty in deriving a numerical optimal bandwidth grows as the dimension of data increases. Another criterion for choosing an optimal bandwidth is the mean integrated squared error (MISE) expressed as

$$
\operatorname{MISE}(H)=E \int_{R^{d}}\left[\hat{f}_{H}(\boldsymbol{x})-f(\boldsymbol{x})\right]^{2} d \boldsymbol{x} .
$$

It is well known that the optimal bandwidth that minimizes MISE does not have a closed form. In order to make progress under this criterion, it is usual to employ an asymptotic approximation. When data are observed from the multivariate normal density and the diagonal bandwidth matrix, denoted by $H=\operatorname{diagonal}\left(h_{1}, h_{2}, \cdots, h_{d}\right)$, is employed, the optimal bandwidth that minimizes MISE can be approximated by (Scott, 1992; Bowman and Azzalini, 1997)

$$
h_{i}=\sigma_{i}\left\{\frac{4}{(d+2) n}\right\}^{1 /(d+4)},
$$

for $i=1,2, \ldots, d$, where $\sigma_{i}$ is the standard deviation of the $i$ th variate and can be replaced by its sample estimator in practical implementations. We call this the "normal reference rule". This method is often used in practice, in the absence of any other practical bandwidth selection schemes, despite the fact that most interesting data are non-Gaussian, and that a full bandwidth matrix is preferable. 
Sain, Baggerly and Scott (1994) derived an estimate of the asymptotic MISE (AMISE) for bivariate densities and employed biased cross-validation to estimate the optimal bandwidth. However, their method cannot be directly extended to the general multivariate setting. Wand and Jones (1995) showed that under certain smoothness assumptions on the target density, the AMISE is expressed as

$$
\operatorname{AMISE}(H)=\frac{1}{n}|H|^{-1 / 2} \int_{R^{2}} K^{2}(\boldsymbol{x}) d \boldsymbol{x}+\frac{1}{4} \int_{R^{2}} \boldsymbol{x} \boldsymbol{x}^{\prime} K^{2}(\boldsymbol{x}) d \boldsymbol{x}\left(\operatorname{vech}^{\prime} H\right) \Psi_{4}(\operatorname{vech} H),
$$

where 'vech' is the vector half operator, and $\Psi_{4}$ is a matrix whose elements are functionals of the unknown target density $f(\boldsymbol{x})$. An estimate of the optimal bandwidth can be derived using the plug-in method, which aims to minimize $\operatorname{AMISE}(H)$ by plugging an estimate of $\Psi_{4}$ in the above equation. For bivariate data, Wand and Jones (1994) presented a plug-in algorithm, which requires auxiliary smoothing parameters. The technology for choosing these auxiliary smoothing parameters is not well developed. Duong and Hazelton (2003) argued that the full bandwidth matrix selectors suggested by Wand and Jones (1994) fail to produce plug-in bandwidths for some data sets. In response to this problem, Duong and Hazelton (2003) presented an alternative plug-in algorithm, which has the advantage that it always produces a finite bandwidth matrix and requires computation of fewer pilot bandwidths. However, these plug-in algorithms cannot be directly extended to the general multivariate setting.

The maximum likelihood cross-validation criterion (discussed in Section 21) leads to an optimal bandwidth that minimizes the Kullback-Leibler information. The likelihood cross-validation bandwidth selector requires a numerical optimization procedure, which becomes increasingly difficult to implement as the dimension of data increases (see, for example, Härdle, 1991). However, from a Bayesian perspective, we can treat nonzero 
components of $H$ as parameters, whose posterior density can be obtained through the likelihood cross-validation criterion. A posterior estimate of $H$ can be derived through the MCMC technique. One important advantage of the MCMC technique for estimating optimal bandwidths is that it is applicable to data of any dimension, not only to bivariate data. Moreover, the sampling algorithm involves no increased difficulty as the dimension of the data increases.

To our knowledge, the only previous paper employing a Bayesian approach to bandwidth selection for kernel density estimation is Brewer (2000). He derived adaptive bandwidths for univariate kernel density estimation, treating the bandwidths as parameters and estimating them via MCMC simulations. Brewer (2000) showed that the proposed Bayesian approach is superior to methods of Abramson (1982) and Sain and Scott (1996).

Schuster and Gregory (1981) demonstrated that in some circumstances, likelihood cross-validation produces inconsistent estimates for univariate kernel density estimation. However, Brewer (2000) argued that the MCMC approach to adaptive bandwidth selection may avoid the inconsistency problem by choosing an appropriate prior and using a kernel with infinite support. The same argument applies to the case considered here.

In this paper, we present MCMC algorithms for estimating the optimal bandwidth matrix for multivariate kernel density estimation through the likelihood cross-validation criterion, and sampling algorithms are developed for both diagonal and full bandwidth matrices. The rest of this paper is organized as follows. Section 2 briefly discusses the likelihood cross-validation criterion and presents MCMC algorithms for both diagonal and full bandwidth matrices. In Section 3, we examine the performance of MCMC algorithms 
with data generated from known bivariate densities. We find that the MCMC algorithm generally performs better than either the plug-in algorithm or the normal reference rule in the bivariate setting. Section 4 applies the MCMC bandwidth selectors to data generated from known multivariate densities, and we find that the MCMC algorithm performs much better than the normal reference rule (there are no other bandwidth selection methods available in this case). Section 5 illustrates the use of the MCMC algorithm for bandwidth selection with an application to some earthquake data and to estimation of financial data based on Monte Carlo kernel likelihood. We provide conclusions in Section 6 .

\section{MCMC for optimal bandwidth selection}

\subsection{Likelihood cross-validation}

Kullback-Leibler information is a measure of distance between two densities. Our interest is in choosing the approximate density $\hat{f}_{H}(\boldsymbol{x})$ to minimize its distance from the target density $f(\boldsymbol{x})$. In this case, Kullback-Leibler information is defined as

$$
\begin{aligned}
d_{K L}\left(f, \hat{f}_{H}\right) & =\int_{R^{d}} \log \left[\frac{f(\boldsymbol{x})}{\hat{f}_{H}(\boldsymbol{x})}\right] f(\boldsymbol{x}) d \boldsymbol{x} \\
& =\int_{R^{d}} \log [f(\boldsymbol{x})] f(\boldsymbol{x}) d \boldsymbol{x}-\int_{R^{d}} \log \left[\hat{f}_{H}(\boldsymbol{x})\right] f(\boldsymbol{x}) d \boldsymbol{x},
\end{aligned}
$$

which is nonnegative. We want to find an optimal bandwidth that minimizes $d_{K L}\left(f, \hat{f}_{H}\right)$, or, equivalently, maximizes

$$
\mathrm{E} \log \left[\hat{f}_{H}(\boldsymbol{x})\right]=\int_{R^{d}} \log \left[\hat{f}_{H}(\boldsymbol{x})\right] f(\boldsymbol{x}) d \boldsymbol{x},
$$

which can be approximated by

$$
\hat{\mathrm{E}} \log \left[\hat{f}_{H}(\boldsymbol{x})\right]=\sum_{i=1}^{n} \log \hat{f}_{H}\left(\boldsymbol{x}_{i}\right)=\sum_{i=1}^{n} \log \left[\frac{1}{n} \sum_{j=1}^{n} K_{H}\left(\boldsymbol{x}_{\boldsymbol{i}}-\boldsymbol{x}_{j}\right)\right] .
$$


If we directly maximize (2) with respect to $H$, the resulting bandwidth is a matrix of zeros. A way out of this dilemma is to estimate $f_{H}\left(\boldsymbol{x}_{i}\right)$ based on the subset $\left\{\boldsymbol{x}_{j}: j \neq i\right\}$, and to approximate $\mathrm{E} \log \left[\hat{f}_{H}(\boldsymbol{x})\right]$ by (Härdle, 1991)

$$
L\left(\boldsymbol{x}_{1}, \boldsymbol{x}_{2}, \ldots, \boldsymbol{x}_{n} \mid H\right)=\sum_{i=1}^{n} \log \hat{f}_{H, i}\left(\boldsymbol{x}_{i}\right)
$$

where $\hat{f}_{H, i}$ is the leave-one-out estimator

$$
\hat{f}_{H, i}\left(\boldsymbol{x}_{i}\right)=\frac{1}{n-1} \sum_{\substack{j=1 \\ j \neq i}}^{n}|H|^{-1 / 2} K\left(H^{-1 / 2}\left(\boldsymbol{x}_{i}-\boldsymbol{x}_{j}\right)\right) .
$$

The likelihood cross-validation criterion is to select $H$ by maximizing $n^{-1} L(\cdot \mid H)$.

Solving this maximization problem requires a numerical procedure, which becomes increasingly difficult to implement as the dimension increases. However, when nonzero components of $H$ are treated as parameters, the logarithmic likelihood of $\left\{\boldsymbol{x}_{1}, \boldsymbol{x}_{2}, \cdots, \boldsymbol{x}_{n}\right\}$ is provided by (3), and the posterior density of the parameters is proportional to the product of the joint prior density of nonzero components of $H$ and the likelihood. As the MCMC technique is very powerful in sampling a high dimensional vector of parameters, it can be employed to obtain a posterior estimate for the bandwidth matrix.

It is worth noting that rather than the MISE criterion and Kullback-Leibler information criterion for optimal bandwidth selection, one can use the criterion of maximizing accuracy of the mode location. However, under this criterion, it is impossible to obtain the likelihood of $\left\{\boldsymbol{x}_{1}, \boldsymbol{x}_{2}, \cdots, \boldsymbol{x}_{n}\right\}$ given nonzero bandwidths as parameters. In contrast, Kullback-Leibler information provides the possibility of deriving the likelihood, which we use to construct the posterior density. 


\subsection{Sampling a diagonal bandwidth matrix}

When $H$ is diagonal, the kernel density estimator of $f(\boldsymbol{x})$ is

$$
\hat{f}_{\boldsymbol{h}}(\boldsymbol{x})=\frac{1}{n} \sum_{j=1}^{n} \frac{1}{h_{1} h_{2} \cdots h_{d}} K\left(\frac{x_{1}-x_{j, 1}}{h_{1}}, \frac{x_{2}-x_{j, 2}}{h_{2}}, \cdots, \frac{x_{d}-x_{j, d}}{h_{d}}\right)
$$

where $\boldsymbol{h}=\left(h_{1}, h_{2}, \cdots, h_{d}\right)^{\prime}$ is a vector of bandwidths with positive values. The leave-oneout estimator is

$$
\hat{f}_{\boldsymbol{h}, i}\left(\boldsymbol{x}_{i}\right)=\frac{1}{n-1} \sum_{\substack{j=1 \\ j \neq i}}^{n} \frac{1}{h_{1} h_{2} \cdots h_{d}} K\left(\frac{x_{i, 1}-x_{j, 1}}{h_{1}}, \frac{x_{i, 2}-x_{j, 2}}{h_{2}}, \cdots, \frac{x_{i, d}-x_{j, d}}{h_{d}}\right),
$$

for $i=1,2, \cdots, n$. We treat the bandwidth $\boldsymbol{h}$ as a vector of parameters, given which, the likelihood function of $\left\{\boldsymbol{x}_{1}, \boldsymbol{x}_{2}, \cdots, \boldsymbol{x}_{n}\right\}$ is

$$
L\left(\boldsymbol{x}_{1}, \boldsymbol{x}_{2}, \cdots, \boldsymbol{x}_{n} \mid \boldsymbol{h}\right)=\sum_{i=1}^{n} \log \hat{f}_{\boldsymbol{h}, i}\left(\boldsymbol{x}_{i}\right)
$$

We assume that the prior density of each component of $\boldsymbol{h}$ is (up to a normalizing constant)

$$
\pi\left(h_{k} \mid \lambda\right) \propto \frac{1}{1+\lambda h_{k}^{2}}
$$

for $k=1,2, \cdots, d$, where $\lambda$ is a hyperparameter controlling the shape of the prior density. According to Bayes theorem, the posterior of $\boldsymbol{h}$ is (up to a normalizing constant)

$$
\pi\left(\boldsymbol{h} \mid \boldsymbol{x}_{1}, \boldsymbol{x}_{2}, \cdots, \boldsymbol{x}_{n}\right) \propto\left[\prod_{k=1}^{d} \frac{1}{1+\lambda h_{k}^{2}}\right] \times \prod_{i=1}^{n} \hat{f}_{\boldsymbol{h}, i}\left(\boldsymbol{x}_{i}\right),
$$

from which we can sample $\boldsymbol{h}$ using the Metropolis-Hastings algorithm. The ergodic average or the posterior mean of $\boldsymbol{h}$ acts as an estimator of optimal bandwidth.

The likelihood appearing in the posterior density given by (4) is flat when components of $\boldsymbol{h}$ are large. If we use uniform priors for the components of $\boldsymbol{h}$ and employ the randomwalk Metropolis-Hastings algorithm to sample $\boldsymbol{h}$, the update of $\boldsymbol{h}$ has a negligible effect 
when components of $\boldsymbol{h}$ are already very large. In order to make the sampling algorithm work appropriately, sufficient prior information on components of $\boldsymbol{h}$ is required to put a low prior probability on the "problematic" region in the parameter space, where the likelihood function is flat. In this sense, the effect of the prior given by (5) seems to be a penalty on the likelihood.

In a different context, Bauwens and Lubrano (1998) used a similar prior for the degreesof-freedom parameter of the $t$-distribution. They proved that with a diffuse prior on the degrees-of-freedom parameter on $(0, \infty)$, the resulting posterior density is not integrable, and a prior of the form of (5) provides integrability. In our case, we can show that a diffuse prior on each component of $\boldsymbol{h}$ results in a posterior that is integrable on $(0, \infty)$. Hence the purpose of the prior given by (5) is not to provide integrability but to make the sampling algorithm work appropriately.

As well as the leave-one-out method, there are some other cross-validation methods, such as the fixed-fraction version cross-validation discussed by van der Laan, Dudoit and Keles (2004). They showed that the fixed-fraction cross-validation method has good asymptotic properties for model selection. The fixed-fraction cross-validation is generally appropriate for the proposed Bayesian framework for choosing an optimal bandwidth.

\subsection{Sampling a full bandwidth matrix}

As the bandwidth matrix is symmetric positive definite, we can obtain its Cholesky de-

composition $H=L L^{\prime}$, where $L$ is a lower triangular matrix. Let $B=L^{-1}$ which is also 
lower triangular. Then the kernel estimator of $f(\boldsymbol{x})$ is

$$
\hat{f}_{B}(\boldsymbol{x})=\frac{1}{n}|B| \sum_{i=1}^{n} K\left(B\left(\boldsymbol{x}-\boldsymbol{x}_{i}\right)\right)
$$

and the leave-one-out estimator of $f(\boldsymbol{x})$ is

$$
\hat{f}_{B, i}\left(\boldsymbol{x}_{i}\right)=\frac{1}{n-1}|B| \sum_{\substack{j=1 \\ j \neq i}}^{n} K\left(B\left(\boldsymbol{x}_{i}-\boldsymbol{x}_{j}\right)\right) .
$$

We treat non-zero elements of the bandwidth matrix as parameters, whose posterior density can be obtained based on the likelihood function given in (3). We assume that the prior density of each non-zero component of $B$ is (up to a normalizing constant)

$$
\pi\left(b_{i j} \mid \lambda\right) \propto \frac{1}{1+\lambda b_{i j}^{2}}
$$

for $j \leq i$ and $i=1,2, \ldots, d$. Using Bayes theorem, we can obtain the posterior density of $B$ (up to a normalizing constant)

$$
\pi\left(B \mid \boldsymbol{x}_{1}, \boldsymbol{x}_{2}, \cdots, \boldsymbol{x}_{n}\right) \propto\left[\prod_{i=1}^{d} \prod_{j=1}^{i} \frac{1}{1+\lambda b_{i j}^{2}}\right] \times \prod_{i=1}^{n} \hat{f}_{B, i}\left(\boldsymbol{x}_{i}\right),
$$

from which we sample all elements of $B$ using the Metropolis-Hastings algorithm. The ergodic average or the posterior mean of $B$ acts as an estimator of optimal bandwidth.

\subsection{Transformation of data}

The plug-in algorithm for bandwidth selection developed by Duong and Hazelton (2003) uses a simple form for the pilot bandwidths, which is inappropriate when the dispersion of the data differs markedly between the two variates. Hence Duong and Hazelton (2003) suggested that the data be pre-scaled before the plug-in algorithm is implemented. 
Given a set of bivariate data denoted by $\left\{\boldsymbol{x}_{1}, \boldsymbol{x}_{2}, \ldots, \boldsymbol{x}_{n}\right\}$, let $S$ denote the sample variance-covariance matrix with diagonal components $s_{1}^{2}$ and $s_{2}^{2}$. Duong and Hazelton (2003) defined the sphering and scaling transformations, respectively, by

$$
\boldsymbol{x}_{i}^{*}=S^{-1 / 2} \boldsymbol{x}_{i}, \quad \text { and } \quad \boldsymbol{x}_{i}^{*}=S_{d}^{-1 / 2} \boldsymbol{x}_{i}
$$

for $i=1,2, \ldots, n$, where $S_{d}=\operatorname{diagonal}\left(s_{1}^{2}, s_{2}^{2}\right)$. When the optimal bandwidth matrix, denoted by $\hat{H}^{*}$, for the transformed data is obtained, the optimal bandwidth matrix for the original data can be calculated through the reverse transformation, $\hat{H}=S^{1 / 2} \hat{H}^{*}\left(S^{1 / 2}\right)^{\prime}$ or $\hat{H}=S_{d}^{1 / 2} \hat{H}^{*} S_{d}^{1 / 2}$.

To sample a bandwidth matrix, we shall use the random-walk Metropolis-Hastings algorithm, in which scaling (and possibly sphering) is of prime importance because the algorithm has to mix different scales of different variates (and to incorporate correlations between variates). This kind of scaling (and sphering) is incorporated in the proposal density and is different from the scaling and sphering pre-transformations of the data defined in (9). If we make a scaling or sphering pre-transformation of the data, for which we derive an estimate of the optimal bandwidth, then we have to make a reverse transformation to derive an estimated bandwidth for the original data. However, the sampling algorithm can directly produce an estimated bandwidth for the original data, even though a certain kind of scaling and sphering might be involved.

If we choose a sphering transformation of data and use the diagonal bandwidth matrix, the resulting bandwidth estimator for the original data is a full matrix. When the variates are correlated and the diagonal bandwidth matrix is used, the bandwidth matrix estimator obtained through the sphering transformation of the original data might produce a better performance than that obtained directly from the original data, because the sphering 
transformation is equivalent to pre-rotating the data (see, for example, Wand and Jones 1993).

\section{Numerical studies with bivariate densities}

This section examines the performance of the proposed MCMC methods for bandwidth selection via several sets of bivariate data, generated from known densities. As the true density is known in each case, the performance of the bandwidth can be measured by the accuracy of the corresponding kernel density estimator via Kullback-Leibler information.

Kullback-Leibler information defined in (1) is the mean of $\log \left(f(\boldsymbol{x}) / \hat{f}_{H}(\boldsymbol{x})\right)$ under density $f(\boldsymbol{x})$, and so it measures the discrepancy of the estimated density from the true density. If a large number of random vectors, denoted by $\left\{\boldsymbol{x}_{1}, \boldsymbol{x}_{2}, \ldots, \boldsymbol{x}_{N}\right\}$, can be drawn from $f(\boldsymbol{x})$, Kullback-Leibler information can be estimated by

$$
\hat{d}_{K L}\left(f, \hat{f}_{H}\right)=\frac{1}{N} \sum_{i=1}^{N} \log \left(f\left(\boldsymbol{x}_{i}\right) / \hat{f}_{H}\left(\boldsymbol{x}_{i}\right)\right) .
$$

\subsection{True densities}

We consider four target densities labelled A, B, C and D, respectively. Contour plots of these densities are shown in Figure 1, Density A is a mixture of two bivariate normal densities, with high correlation and bimodality:

$$
f_{A}\left(\boldsymbol{x} \mid \mu_{1}, \Sigma_{1}, \mu_{2}, \Sigma_{2}\right)=\frac{1}{2} \phi\left(\boldsymbol{x} \mid \mu_{1}, \Sigma_{1}\right)+\frac{1}{2} \phi\left(\boldsymbol{x} \mid \mu_{2}, \Sigma_{2}\right),
$$


where $\phi(\boldsymbol{x} \mid \mu, \Sigma)$ denotes a multivariate normal density with mean $\mu$ and variancecovariance matrix $\Sigma$, and

$$
\mu_{1}=\left(\begin{array}{l}
2 \\
2
\end{array}\right), \quad \Sigma_{1}=\left(\begin{array}{rr}
1 & -0.9 \\
-0.9 & 1
\end{array}\right), \quad \mu_{2}=\left(\begin{array}{l}
-1.5 \\
-1.5
\end{array}\right), \quad \Sigma_{2}=\left(\begin{array}{rr}
1 & 0.3 \\
0.3 & 1
\end{array}\right)
$$

Density B is a bivariate skew-normal density with high correlation:

$$
f_{B}(\boldsymbol{x} \mid \mu, \Sigma, \alpha)=2 \phi(\boldsymbol{x} \mid \mu, \Sigma) \Phi\left(\alpha^{\prime} w^{-1 / 2}(\boldsymbol{x}-\mu)\right)
$$

where $\Phi(\cdot)$ is the cumulative density function of a standard bivariate normal distribution, and $w$ is a diagonal matrix with diagonal elements the same as those of $\Sigma$. This distribution has been studied by Azzalini and Dalla Valle (1996), Azzalini and Capitanio (1999, 2003), Jones (2001) and Jones and Faddy (2003) among others. Here $\alpha$ is a shape parameter capturing the skewness. When $\alpha=0$, this density becomes the usual normal density. For the purpose of generating a set of data, we use the following parameters,

$$
\mu=\left(\begin{array}{l}
2 \\
2
\end{array}\right), \quad \Sigma=\left(\begin{array}{rr}
1 & 0.9 \\
0.9 & 1
\end{array}\right), \quad \alpha=\left(\begin{array}{l}
0.5 \\
0.5
\end{array}\right)
$$

Density $\mathrm{C}$ is a mixture of two bivariate Student $t$ densities:

$$
f_{C}\left(\boldsymbol{x} \mid \mu_{1}, \mu_{2}, \Sigma, \nu\right)=\frac{1}{2} t_{d}\left(\boldsymbol{x} \mid \mu_{1}, \Sigma, \nu\right)+\frac{1}{2} t_{d}\left(\boldsymbol{x} \mid \mu_{2}, \Sigma, \nu\right)
$$

where

$$
t_{d}(\boldsymbol{x} \mid \mu, \Sigma, \nu)=\frac{\Gamma((\nu+d) / 2)}{(\nu \pi)^{d / 2} \Gamma(\nu / 2)|\Sigma|^{1 / 2}}\left[1+\frac{1}{\nu}(\boldsymbol{x}-\mu)^{\prime} \Sigma^{-1}(\boldsymbol{x}-\mu)\right]^{-(d+\nu) / 2},
$$

has location parameter $\mu$, dispersion matrix $\Sigma$ and degrees of freedom $\nu$, and with parameters set to

$$
\mu_{1}=\left(\begin{array}{r}
-1.5 \\
0
\end{array}\right), \quad \mu_{2}=\left(\begin{array}{r}
1.5 \\
0
\end{array}\right), \quad \Sigma=\left(\begin{array}{rr}
1 & 0.9 \\
0.9 & 1
\end{array}\right)
$$


and $\nu=5$. Density $\mathrm{C}$ exhibits heavy tail behaviour, high correlation and bimodality.

Density D is a mixture of two bivariate Student $t$ densities, but has thicker tails than density D:

$$
f_{D}\left(\boldsymbol{x} \mid \mu_{1}, \mu_{2}, \Sigma, \nu\right)=\frac{1}{2} t_{d}\left(\boldsymbol{x} \mid \mu_{1}, \Sigma_{1}, \nu\right)+\frac{1}{2} t_{d}\left(\boldsymbol{x} \mid \mu_{2}, \Sigma_{2}, \nu\right),
$$

where $\nu=3$,

$$
\mu_{1}=\left(\begin{array}{l}
3 \\
3
\end{array}\right), \quad \Sigma_{1}=\left(\begin{array}{rr}
1 & 0.75 \\
0.75 & 1
\end{array}\right), \quad \mu_{2}=\left(\begin{array}{l}
-3 \\
-3
\end{array}\right), \quad \text { and } \quad \Sigma_{2}=\left(\begin{array}{rr}
1 & 0.5 \\
0.5 & 1
\end{array}\right)
$$

\subsection{Bandwidth matrix selectors}

From each of the proposed bivariate densities, we generate data sets of size $n=200$, 500 and 1000, respectively. For each data set, we calculate the bivariate kernel density estimator using the standard bivariate Gaussian kernel function and bandwidth matrix selected through each of the following selectors.

$M_{1}$ : MCMC algorithm for full bandwidth matrix without pre-transformation of data; $M_{2}$ : MCMC algorithm for full bandwidth matrix with scaling transformation of data; $M_{3}$ : MCMC algorithm for full bandwidth matrix with sphering transformation of data; $M_{4}$ : MCMC algorithm for diagonal bandwidth matrix without pre-transformation; $M_{5}:$ MCMC algorithm for diagonal bandwidth matrix with scaled data; $M_{6}:$ MCMC algorithm for diagonal bandwidth matrix with sphered data; $P_{1}$ : Plug-in selector of full bandwidth matrix with scaling transformation of data; $P_{2}$ : Plug-in selector of full bandwidth matrix with sphering transformation of data; $P_{3}$ : Plug-in selector of diagonal bandwidth matrix with scaling transformation of data; 
$P_{4}$ : Plug-in selector of diagonal bandwidth matrix with sphering transformation of data; $N_{1}$ : The normal reference rule approach for a diagonal bandwidth.

The plug-in bandwidth selector refers to the algorithm developed by Duong and Hazelton (2003). We have not included the plug-in algorithms of Wand and Jones (1993), because their algorithm for full bandwidth matrix selection sometimes fails to produce finite bandwidths for some data sets. When their algorithm works, its performance is similar to the plug-in algorithm developed by Duong and Hazelton (2003). See Duong and Hazelton (2003) for further discussion of these two plug-in algorithms.

\subsection{MCMC outputs and sensitivity analysis}

The hyperparameter of prior densities defined in (7) is initially set to $\lambda=1$ which represents a very flat prior. Given a data set generated from a bivariate density, we sample the diagonal and full bandwidth matrices from their corresponding posterior densities defined in (88) using the random-walk Metropolis-Hastings algorithm, in which the proposal density is the multivariate standard normal density. In order to prevent a false impression of convergence, the tuning parameter was chosen so that the acceptance rate was between 0.2 and 0.3 .

The burn-in period is set at 5,000 iterations, and the number of total recorded iterations is 25,000 . The initial value of $B$ is set to the identity matrix. After we obtain the sampled path of $B$ for each data set, we calculate the ergodic average (or posterior mean) and the batch-mean standard error (see, for example, Roberts 1996), where the number of batches is 50 and there are 500 draws in each batch. The ergodic average acts as an estimator of optimal bandwidth. 
We ran our sampling algorithms on a parallel unix system, whose processor is a 64-bit EV6.8AL with $834 \mathrm{MHz}$ and $2 \mathrm{G}$ RAM. The required CPU time is around 2 minutes for a sample size of 200, 15 minutes for a sample size of 500, and 55 minutes for a sample size of 1000. There is no obvious difference in computing time between the full bandwidth matrix sampler and the diagonal bandwidth matrix sampler (see Table 8).

We used the batch-mean standard error and the simulation inefficiency factor (SIF) to check the mixing performance of the sampling algorithm (see, for example, Roberts, 1996; Kim, Shephard and Chib, 1998; Tse, Zhang and Yu, 2004). We use $f_{D}(\cdot)$ as an example to illustrate the mixing performance of the sampling algorithm. Table 1 presents a summary of MCMC outputs obtained through $M_{1}$ and $M_{6}$. Both SIF and the batch-mean standard error show that all the simulated chains have mixed very well. We found a similar mixing performance for the other sampling algorithms, and for the other data sets.

We examined the robustness of the results to prior choices by trying values of $\lambda=0.1$ and $\lambda=5$, as well as $\lambda=1$. The mixing performance and posterior mean of each sampler was similar in all cases.

\subsection{Accuracy of MCMC bandwidth selectors}

In order to estimate the Kullback-Leibler information, we generated $N=100,000$ bivariate random vectors from the true density and calculated the estimated Kullback-Leibler information defined by (10), which is employed to measure the distance between the bivariate kernel density estimator and the corresponding true density. Table 2 presents the estimated Kullback-Leibler information for each density and each bandwidth selector. 
The simulation study reveals the following evidence.

- For data sets generated from $f_{C}$ and $f_{D}$, the MCMC bandwidth selector performs better than the corresponding plug-in bandwidth selector; for data sets generated from $f_{A}$, both selectors have a similar performance; for data sets generated from $f_{B}$, the MCMC bandwidth selector performs better than the plug-in bandwidth selector except when using a sphering transformation for a full bandwidth matrix.

- For each data set generated, the MCMC bandwidth selector performs better than the normal reference rule.

- The scaling transformation adds nothing to the performance of MCMC algorithms for sampling both diagonal and full bandwidth matrices.

- The sphering transformation of data is only helpful to the MCMC algorithm for sampling a diagonal bandwidth matrix when two variates are correlated, such as for densities B and D. For uncorrelated data, and for sampling a full bandwidth matrix, sphering can degrade performance. This is also supported by Wand and Jones (1993).

- The MCMC algorithm for a diagonal bandwidth matrix applied after sphering does not perform quite as well as the full bandwidth approach. However, the simplicity of using a diagonal bandwidth matrix makes this an attractive approach, especially with high dimensional data.

It seems reasonable to interpret the choice between diagonal and full bandwidth matrices as a bias-variance tradeoff between diagonal and full covariance matrices, because there are more parameters in a full bandwidth matrix than in a diagonal matrix. For 
high dimensional data, one might prefer more biased, lower variance estimates of a diagonal matrix over less biased but highly variable estimates of a full bandwidth matrix. Also sample size has a role to play in this choice - the larger the sample, the greater the confidence we can have in estimating the full set of parameters. When sphering is necessary, we found that the performance of a full bandwidth matrix is poorer than that of a diagonal bandwidth matrix (as indicated by $f_{B}$ ).

We also employed the MISE criterion to examine the performance of optimal bandwidths obtained through the MCMC algorithm, the bivariate plug-in algorithm and the normal reference rule. We computed numerical MISEs for algorithms $M_{6}, P_{4}$ and $N_{1}$ through 50 data sets of sample sizes 200, 500 and 1000, each of which was generated from $f_{D}(\cdot)$. Results are given in the second column of Table 3, which shows that $M_{6}$ performs slightly better than $P_{4}$ for sample size 200 , and slightly poorer than $P_{4}$ for sample sizes 500 and 1000.

When one bandwidth selector has a lower MISE than another method, it is useful to look at the standard deviation of the integrated squared errors (ISE). $M_{6}$ has less bias and larger variations than $P_{4}$ for sample size 200, while for sample sizes 500 and 1000, both bias and variation of $M_{6}$ are larger than those of $P_{4}$. In addition, both bandwidth selectors have less bias and larger variations than the normal reference rule.

We also computed the average difference between the ISEs of any two bandwidth selectors. The difference in ISE between $M_{6}$ and $P_{4}$ is insignificant, but the difference in ISE between $M_{6}$ and $N_{1}$, as well as that between $P_{4}$ and $N_{1}$, are significant. Both $M_{6}$ and $P_{4}$ perform significantly better than $N_{1}$. Hence the empirical experience shows that 
$M_{6}$ and $P_{4}$ have a similar accuracy while $M_{6}$ is more variable that $P_{4}$, and that both $M_{6}$ and $P_{4}$ are significantly less biased and more variable than $N_{1}$. As the computation of numerical MISE is time-consuming, we have not computed MISE for the other bandwidth selectors, and for data sets generated from the other densities.

\section{Numerical studies with multivariate densities}

In this section, we examine the accuracy of the MCMC approach in the general multivariate setting. Our examples use $d=5$.

\subsection{True densities and bandwidth selectors}

We consider five target densities labelled E, F, G, H and I, respectively. Density E is a multivariate normal density with location parameter $\mu$ and variance-covariance matrix defined as

$$
\Sigma=\frac{1}{1-\rho^{2}}\left(\begin{array}{ccccc}
1 & \rho & \rho^{2} & \rho^{3} & \rho^{4} \\
\rho & 1 & \rho & \rho^{2} & \rho^{3} \\
\rho^{2} & \rho & 1 & \rho & \rho^{2} \\
\rho^{3} & \rho^{2} & \rho & 1 & \rho \\
\rho^{4} & \rho^{3} & \rho^{2} & \rho & 1
\end{array}\right)
$$

where $\rho=0.9$ and $\mu=(2,2,2,2,2)^{\prime}$. This density is produced by a first order autoregressive linear time series model.

Density $\mathrm{F}$ is a mixture of two multivariate normal densities,

$$
f_{F}\left(\boldsymbol{x} \mid \mu_{1}, \mu_{2}, \Sigma\right)=\frac{1}{2} \phi\left(\boldsymbol{x} \mid \mu_{1}, \Sigma\right)+\frac{1}{2} \phi\left(\boldsymbol{x} \mid \mu_{2}, \Sigma\right)
$$

where $\mu_{1}=(2,2,2,2,2)^{\prime}, \mu_{2}=(-1.5,-1.5,-1.5,-1.5,-1.5)^{\prime}$ and $\Sigma$ is the $5 \times 5$ identity matrix. 
Density G is a mixture of two multivariate Student $t$ densities,

$$
f_{G}\left(\boldsymbol{x} \mid \mu_{1}, \mu_{2}, \Sigma, \nu\right)=\frac{1}{2} t_{d}\left(\boldsymbol{x} \mid \mu_{1}, \Sigma, \nu\right)+\frac{1}{2} t_{d}\left(\boldsymbol{x} \mid \mu_{2}, \Sigma, \nu\right)
$$

with $t_{d}(\cdot)$ defined in (11) $, \mu_{1}=(2,2,2,2,2)^{\prime}, \mu_{2}=(-1.5,-1.5,-1.5,-1.5,-1.5)^{\prime}, \Sigma$ is the identity matrix, and $\nu=3$.

Density $\mathrm{H}$ is the multivariate skew normal density,

$$
f_{H}(\boldsymbol{x} \mid \mu, \Sigma, \alpha)=2 \phi(\boldsymbol{x} \mid \mu, \Sigma) \Phi\left(\alpha^{\prime} w^{-1 / 2}(\boldsymbol{x}-\mu)\right),
$$

where $\Phi(\cdot)$ is the cumulative density function of a standard multivariate normal distribution, and $w$ is a diagonal matrix with diagonal elements the same as those of $\Sigma$. To generate a set of data, we define these parameters as $\mu=(2,2,2,2,2)^{\prime}, \Sigma$ as (12) with $\rho=0.9$, and skewness parameter vector $\alpha=(-0.5,-0.5,-0.5,-0.5,-0.5)^{\prime}$.

Density I is the multivariate skew $t$ density,

$$
f_{I}(\boldsymbol{x} \mid \mu, \Sigma, \nu, \alpha)=2 t_{d}(\boldsymbol{x} \mid \mu, \Sigma, \nu) T_{d}(\tilde{\boldsymbol{x}} \mid \nu+d)
$$

where $t_{d}(\cdot)$ is the multivariate $t$ density defined in (11), $T_{d}(\cdot \mid \nu+d)$ is the cumulative density function of a multivariate $t$ distribution with mean $\mathbf{0}$, identity dispersion matrix and degrees of freedom $\nu+d$, and

$$
\tilde{\boldsymbol{x}}=\alpha^{\prime} w^{-1 / 2}(\boldsymbol{x}-\mu)\left(\frac{\nu+d}{(\boldsymbol{x}-\mu)^{\prime} \Sigma^{-1}(\boldsymbol{x}-\mu)+\nu}\right)^{1 / 2},
$$

with $w$ the diagonal matrix with diagonal elements the same as those of $\Sigma$.

From each of the proposed multivariate densities, we generated data sets of sizes 500, 1000 and 1500. Then we applied the proposed MCMC algorithms to each data set to estimate the optimal bandwidth, where the multivariate standard Gaussian kernel is used. 
As the normal reference rule discussed in Scott (1992) and Bowman and Azzalini (1997) is the only viable alternative, we shall compare the performance of MCMC bandwidth selectors $M_{1}$ to $M_{6}$ with that of the alternative bandwidth selector $N_{1}$. The MCMC algorithm and parameter settings are the same as those in bivariate examples.

\subsection{MCMC outputs and sensitivity analysis}

Table 4 shows MCMC output obtained from $f_{E}(\cdot)$ with size 1500 to illustrate the mixing performance of the sampling algorithm. Both the batch-mean standard error and SIF show that all the sampled chains have mixed very well.

The numerical study shows that all algorithms for a diagonal bandwidth matrix have a similar mixing performance, and that all algorithms for a full bandwidth matrix have a similar mixing performance. However, the algorithm for a diagonal bandwidth matrix usually has a better mixing performance than that for a full bandwidth matrix. Similar results were found for the other data sets. Again, we found that the MCMC results are insensitive to changes in $\lambda$.

\subsection{Accuracy of MCMC bandwidth selectors}

To estimate the Kullback-Leibler information, we generated $N=100,000$ random vectors from the true density and calculated the estimated Kullback-Leibler information defined by (10). Table 5 presents these results for each density and each bandwidth selector.

The simulation study reveals the following evidence. First, all MCMC bandwidth selectors perform much better than the normal reference rule. Second, the scaling trans- 
formation adds nothing to the performance of MCMC algorithms for either the diagonal or full matrices. Third, the sphering transformation of data is only useful for the diagonal bandwidth matrix when variables are correlated (such as with densities $\mathrm{E}, \mathrm{H}$ and I). When there is no correlation, or with the full bandwidth matrix, sphering degrades performance.

As we did in the bivariate case, we employed the MISE criterion to compare the performance of optimal bandwidths obtained through the MCMC algorithm and the normal reference rule. We computed numerical MISEs for algorithms $M_{6}$ and $N_{1}$ through 50 data sets of sample size 500, 1000 and 1500 , each of which was generated from $f_{H}(\cdot)$. The ISE obtained through $M_{6}$ is less than that obtained through $N_{1}$ for every data set. A summary of numerical ISEs is given in Table 6, which shows that the average difference between ISEs of $M_{6}$ and $N_{1}$ is highly significant. As the numerical MISE is computationally intensive, we have not computed MISEs for the other bandwidth selectors, and for data sets generated from the other densities.

The CPU time required by the sampling algorithm (under the same conditions described in Section 3.3) for a diagonal bandwidth matrix is 19 minutes for a sample size of 500, 77 minutes for a sample size of 1000, and 177 minutes for a sample size of 1500 . The computing time required by the sampling algorithm for a full bandwidth matrix is 26 minutes for a sample size of 500, 102 minutes for a sample size of 1000, and 238 minutes for a sample size of 1500 (see Table 8). 


\section{Applications of MCMC bandwidth selectors}

\subsection{An application to earthquake data}

We now apply the methodology to a trivariate data set discussed in Scott (1992). These data represent the epicenters of 510 earthquake tremors that occurred beneath the Mt St Helens volcano in the two months leading up to its eruption in March 1982. The three variables represent latitude, longitude and log-depth below the surface. Scott (1992, plate 8) gave several contours of a kernel density estimate of these data, where the bandwidths appear to have been chosen subjectively. We repeat this plot with the optimal bandwidth computed through our method.

We used the MCMC algorithms $M_{1}$ and $M_{5}$ to obtain optimal bandwidths, where the hyperparameter $\lambda=1$, the burn-in period consists of 5,000 iterations, and the recorded period contains 25,000 iterations. Table 7 tabulates a summary of results. Both the batch-mean standard error and SIF show that all sampled chains have mixed very well.

Using the estimated diagonal bandwidth matrix, we computed a kernel density estimator. (The estimate using the full bandwidth matrix was almost identical in this case.) The $98 \%$ highest density region (Hyndman, 1996) is plotted in Figure 2, The surface was computed using the algorithm of Amenta, Bern and Kamvysselis (1998). Note that the detached shells represent outliers in the data; the large central shell represents the bulk of the epicenters. The figure clearly shows clustering of the epicenters, revealing structure that was not discovered by Scott (1992) using a subjective bandwidth. It would be interesting to identify the clusters with geological features, although this information is not available to us. As the plug-in bandwidth selectors are only applicable to bivariate data, 
we cannot obtain an optimal bandwidth through this method for comparison purposes.

\subsection{Bandwidth selection for a Monte Carlo kernel likelihood}

A difficulty for likelihood-based analysis such as maximum likelihood estimation and likelihood ratio testing with state-space models is that likelihood calculations require a high-dimensional integration of state variables. Let $\mathbf{y}$ denote a vector of observations and $\theta$ a parameter vector. Let $\theta^{(j)}$ denote the $j$ th recorded draw of $\theta$ during MCMC iterations, for $j=1,2, \cdots, m$. de Valpine (2004) presented a Monte Carlo kernel likelihood (MCKL), which is an importance-sampled kernel estimator of the likelihood (up to a normalizing constant)

$$
\hat{L}_{H}(\theta)=\frac{1}{m} \sum_{j=1}^{m} K_{H}\left(\theta-\theta^{(j)}\right) \frac{1}{p\left(\theta^{(j)}\right)},
$$

where $K_{H}(x)=|H|^{-1 / 2} K\left(H^{-1 / 2} x\right)$ with $K(\cdot)$ being a multivariate kernel function, $H$ a symmetric positive definite matrix, and $p(\cdot)$ the prior of $\theta$. The maximum likelihood estimate (MLE) of $\theta$ can be obtained by maximizing $\hat{L}_{H}(\theta)$ with respect to $\theta$.

The MCKL involves selecting a bandwidth (chosen subjectively in de Valpine, 2004) for the posterior sample $\left\{\theta^{(j)}: j=1,2, \cdots, m\right\}$. de Valpine (2004) indicated that further work on automated bandwidth selection would facilitate the application of MCKL. To obtain an optimal bandwidth, we can use bandwidth selectors proposed in Section 2 ,

Consider the stochastic volatility (SV) model (Jacquier, Polson and Rossi, 2004)

$$
\begin{aligned}
& y_{t}=\exp \left(\alpha_{t} / 2\right) \lambda_{t}^{1 / 2} \varepsilon_{t}, \\
& \alpha_{t+1}=\mu+\phi\left(\alpha_{t}-\mu\right)+\sigma u_{t+1},
\end{aligned}
$$

where $\varepsilon_{t} \sim I N(0,1), u_{t+1} \sim I N(0,1), \operatorname{cov}\left(\varepsilon_{t}, u_{t+1}\right)=\rho$, and $\lambda_{t} \sim I G(\nu / 2, \nu / 2)$, which 
is equivalent to the fact that $\nu / \lambda_{t}$ follows a $\chi^{2}$ distribution with $\nu$ degrees of freedom, and the marginal distribution of $v_{t}=\sqrt{\lambda_{t}} \varepsilon_{t}$ is Student $t$ with $\nu$ degrees of freedom. The parameter vector is $\theta=(\phi, \mu, \rho, \sigma, \nu)^{\prime}$, and the data set consists of 1134 continuously compounded daily returns of the Dow Jones industrial average index from the 1st January 2000 to 30th June 2004, excluding weekends and holidays. We employed the sampling algorithm provided by Zhang and King (2004) to obtain a posterior sample of $\theta$, as well as the posterior average of $\theta$, which is $(19.8826,0.1938,-0.4615,-0.3372,0.9694)^{\prime}$.

To derive the optimal bandwidth for the posterior sample, we employed the algorithm for sampling a diagonal bandwidth matrix with scaling transformation of data discussed in Section 2. The estimated bandwidth is $\boldsymbol{h}=(2.5114,0.0136,0.0444,0.0834,0.0039)^{\prime}$ and is employed in the MCKL. The MLE of $\theta$ is $(21.4499,0.2822,-0.6008,-0.3894,0.864)^{\prime}$, which was obtained by numerically maximizing the MCKL. Even though the normalizing constant of the MCKL is unknown, likelihood-based analysis can be conducted using the particle filter algorithm, which aims to approximate the likelihood at the MLE of $\theta$ (see, for example, Kitagawa, 1996; Kim et al., 1998; Zhang and King, 2004). Using the particle filter, we found that the value of the likelihood computed at the MLE of $\theta$ is -1567.95.

The application of the normal reference rule to the same posterior sample resulted in a bandwidth vector of $(2.5599,0.0116,0.0346,0.0701,0.0044)^{\prime}$, which led to a MLE of $\theta$ of $(21.4499,0.2548,-0.5977,-0.3895,0.8611)^{\prime}$. When the likelihood was evaluated at this estimate using the particle filter, we obtained a likelihood value of -1570.28 . Hence the bandwidth obtained through our MCMC sampler produced a maximum likelihood estimate with a larger maximized likelihood than that calculated via the normal reference rule. 
The application of our bandwidth selector to the MCKL indicates the strength of a computational approach to bandwidth selection for multivariate kernel density estimation, because it is much easier to numerically optimize an objective function than it is to work out the theoretical optimum in this case.

\section{Conclusion}

This paper presents MCMC algorithms to estimate the optimal bandwidth for multivariate kernel density estimation via the likelihood cross-validation criterion. This represents the first data-driven bandwidth selection method for density estimation with more than two variables. Our numerical studies show that the sampling algorithms have a very good performance in achieving convergence of the simulated Markov chains, and are insensitive to prior choices.

Under the Kullback-Leibler information criterion, we have found that the MCMC algorithm generally performs better than the bivariate plug-in algorithm of Duong and Hazelton (2003) and the normal reference rule discussed in Scott (1992) and Bowman and Azzalini (1997). Under the MISE criterion, the MCMC algorithm works as well as Duong and Hazelton's (2003) plug-in algorithm, and both algorithms are superior to the normal reference rule. Under both criteria, our sampling algorithm is superior to the normal reference rule for higher dimensional data. Apart from its performance, the other great advantage of our sampling algorithm is that it is applicable to data of any dimension, although the computing time required does increase as the dimension of data increases. In addition, our bandwidth selector provides a data-driven method for the problem of 
choosing an automated bandwidth for the MCKL — identified by de Valpine (2004) as a gap in literature. The effectiveness of our bandwidth selector in this case has been illustrated through an empirical example.

\section{Acknowledgements}

We wish to thank the Editor, Associate Editor, and referees for their very insightful comments that have substantially improved the paper. We extend our sincere thanks to Faming Liang for sharing his coding skills and resources, David Scott for providing the earthquake data, Tarn Duong and Martin Hazelton for providing their R library to compute bivariate plug-in bandwidths, and the Victorian Partnership for Advanced Computing for computational support. We thank Martin Hazelton, Gael Martin, Mervyn Silvapulle and Dabao Zhang for helpful comments. The second author acknowledges support from the Australian Research Council. Any remaining errors are, of course, ours only.

\section{References}

Abramson, I., 1982. On bandwidth variation in kernel estimates - a square root law. The Annals of Statistics 10, 1217-1223.

Aït-Sahalia, Y., 1996. Testing continuous-time models of the spot interest rate, Review of Financial Studies 9, 385-426.

Aït-Sahalia, Y., Lo, A.W., 1998. Nonparametric estimation of state-price densities implicit in financial asset prices. The Journal of Finance 53, 499-547. 
Amenta, N., Bern, M., Kamvysselis, M., 1998. A new Voronoi-based surface reconstruction algorithm. Proceedings of the 25th Annual Conference on Computer Graphics and Interactive Techniques, 415-421.

Azzalini, A., Capitanio, A., 1999. Statistical applications of the multivariate skew normal distribution. Journal of the Royal Statistical Society Series B 61, 579-602.

Azzalini, A., Capitanio, A., 2003. Distributions generated by perturbation of symmetry with emphasis on a multivariate skew $t$-distribution. Journal of the Royal Statistical Society Series B 66, 367-389.

Azzalini, A., Dalla Valle, A., 1996. The multivariate skew normal distribution. Biometrika $83,715-726$.

Bauwens, L., Lubrano, M., 1998. Bayesian inference on GARCH models using the Gibbs sampler. Econometrics Journal 1, C23-C26.

Bowman, A.W., Azzalini, A., 1997. Applied Smoothing Techniques for Data Analysis. Oxford University Press, London.

Brewer, M.J., 2000. A Bayesian model for local smoothing in kernel density estimation. Statistics and Computing 10, 299-309.

de Valpine, P., 2004. Monte Carlo state-space likelihood by weighted posterior kernel density estimation. Journal of the American Statistical Association 99, 523-536.

Donald, S.G., 1997. Inference concerning the number of factors in a multivariate nonparametric relationship. Econometrica 65, 103-131.

Duong, T., Hazelton, M.L., 2003. Plug-in bandwidth selectors for bivariate kernel density estimation. Journal of Nonparametric Statistics 15, 17-30.

Härdle, W., 1991. Smoothing Techniques with Implementation in S. Springer-Verlag, New 
York.

Hyndman, R.J., 1996. Computing and graphing highest density regions. American Statistician 50, 120-126.

Izenman, A.J., 1991. Recent developments in nonparametric density estimation. Journal of the American Statistical Association 86, 205-224.

Jacquier, E., Polson, N.G., Rossi, P.E., 2004. Bayesian analysis of stochastic volatility models with fat-tails and correlated errors. Journal of Econometrics 122, 185-212.

Jones, M.C., 2001. A skew $t$ distribution. In: Charalambides, C.A., Koutras, M.V., Balakrishnan, N. (Ed.), Probability and Statistical Models with Applications: A Volume in Honor of Theophilos Cacoullos. Chapman \& Hall, London, pp. 269-278.

Jones, M.C., Faddy, M.J., 2003. A skew extension of the t-distribution, with applications. Journal of the Royal Statistical Society Series B 66, 159-174.

Jones, M.C., Marron, J.S., Sheather, S.J., 1996. A brief survey of bandwidth selection for density estimation. Journal of the American Statistical Association 91, 401-407.

Kim, S., Shephard, N., Chib, S., 1998. Stochastic volatility: likelihood inference and comparison with ARCH models. Review of Economic Studies 65, 361-393.

Kitagawa, G., 1996. Monte Carlo filter and smoother for Gaussian nonlinear state space models. Journal of Computational and Graphical Statistics 5, 1-25.

Marron, J.S., 1987. A comparison of cross-validation techniques in density estimation. Annals of Statistics 15, 152-162.

Roberts, G.O., 1996. Markov chain concepts related to sampling algorithms. In: Gilks, W.R., Richardson, S., Spiegelhalter, D.J. (Ed.), Markov Chain Monte Carlo in Practice. Chapman \& Hall, London, pp. 45-57. 
Sain, S.R., Baggerly, K.A., Scott, D.W., 1994. Cross-validation of multivariate densities. Journal of the American Statistical Association 89, 807-817.

Sain, S.R., Scott, D.W., 1996. On locally adaptive density estimation. Journal of the American Statistical Association 91, 1525-1534.

Schuster, E.F., Gregory, C.G., 1981. On the nonconsistency of maximum likelihood nonparametric density estimators. In: Eddy, W.F. (Ed.), Computer Science and Statistics: Proceedings of the 13th Symposium on the Interface. Springer-Verlag, New York, pp. 295-298.

Scott, D.W., 1992. Multivariate Density Estimation: Theory, Practice, and Visualization. John Wiley, New York.

Simonoff, J.S., 1996. Smoothing Methods in Statistics. Springer-Verlag, New York.

Stanton, R., 1997. A nonparametric model of term structure dynamics and the market price of interest rate risk. The Journal of Finance 52, 1973-2002.

Tse, Y.K., Zhang, X., Yu, J., 2004. Estimation of hyperbolic diffusion with Markov chain Monte Carlo simulation. Quantitative Finance 4, 158-169.

van der Laan, M.J., Dudoit, S., Keles, S., 2004. Asymptotic optimality of likelihoodbased cross-validation. Statistical Applications in Genetics and Molecular Biology 4 (1), Article 4.

Wand, M.P., Jones, M.C., 1993. Comparison of smoothing parameterizations in bivariate kernel density estimation. Journal of the American Statistical Association 88, 520-528.

Wand, M.P., Jones, M.C., 1994. Multivariate plug-in bandwidth selection. Computational Statistics 9, 97-116.

Wand, M.P., Jones, M.C., 1995. Kernel Smoothing. Chapman \& Hall, London. 
Zhang, X., King, M.L., 2004. Box-Cox stochastic volatility models with heavy tails and correlated errors. mimeo, Monash University. 
Table 1: $M C M C$ results for data generated from $f_{D}(\cdot)$. The first panel is obtained through the algorithm for a diagonal bandwidth matrix $\left(M_{6}\right)$, while the second panel is obtained through the algorithm for a full bandwidth matrix $\left(M_{1}\right)$.

\begin{tabular}{|l|c|r|c|c|r|l|}
\hline $\begin{array}{l}\text { sample } \\
\text { size }\end{array}$ & bandwidths & mean & $\begin{array}{l}\text { standard } \\
\text { deviation }\end{array}$ & $\begin{array}{l}\text { batch-mean } \\
\text { standard error }\end{array}$ & SIF & $\begin{array}{l}\text { acceptance } \\
\text { rate }\end{array}$ \\
\hline 200 & $1 / b_{11}$ & 0.70 & 0.08 & 0.0017 & 10.32 & 0.224 \\
& $1 / b_{22}$ & 0.75 & 0.07 & 0.0015 & 11.77 & \\
\hline 500 & $1 / b_{11}$ & 0.68 & 0.05 & 0.0011 & 11.72 & 0.207 \\
& $1 / b_{22}$ & 0.66 & 0.05 & 0.0009 & 8.73 & \\
\hline 1000 & $1 / b_{11}$ & 0.69 & 0.03 & 0.0006 & 9.83 & 0.216 \\
& $1 / b_{22}$ & 0.61 & 0.03 & 0.0007 & 11.65 & \\
\hline \hline 200 & $b_{11}$ & 1.18 & 0.15 & 0.0035 & 14.48 & 0.245 \\
& $b_{21}$ & -1.38 & 0.34 & 0.0164 & 57.58 & \\
& $b_{22}$ & 1.69 & 0.21 & 0.0098 & 51.78 & \\
\hline 500 & $b_{11}$ & 1.10 & 0.08 & 0.0016 & 11.41 & 0.265 \\
& $b_{21}$ & -1.58 & 0.27 & 0.0137 & 65.54 & \\
& $b_{22}$ & 1.91 & 0.19 & 0.1920 & 52.87 & \\
\hline 1000 & $b_{11}$ & 1.27 & 0.07 & 0.0015 & 11.68 & 0.267 \\
& $b_{21}$ & -0.79 & 0.11 & 0.0028 & 16.02 & \\
& $b_{22}$ & 1.61 & 0.08 & 0.0016 & 9.45 & \\
\hline
\end{tabular}

Table 2: Estimated Kullback-Leibler information for bivariate densities.

\begin{tabular}{|l|r|ccccccccccc|}
\hline & sample & \multicolumn{10}{|c|}{ Kullback-Leibler information } \\
& size & $M_{1}$ & $M_{2}$ & $M_{3}$ & $M_{4}$ & $M_{5}$ & $M_{6}$ & $P_{1}$ & $P_{2}$ & $P_{3}$ & $P_{4}$ & $N_{1}$ \\
\hline$\hat{E}\left(\ln f_{A}\right)=$ & 200 & 0.131 & 0.129 & 0.158 & 0.154 & 0.154 & 0.228 & 0.129 & 0.213 & 0.153 & 0.192 & 0.375 \\
-3.099 & 500 & 0.074 & 0.075 & 0.091 & 0.094 & 0.094 & 0.150 & 0.075 & 0.124 & 0.093 & 0.112 & 0.284 \\
& 1000 & 0.042 & 0.042 & 0.054 & 0.058 & 0.058 & 0.095 & 0.040 & 0.067 & 0.056 & 0.067 & 0.235 \\
$\hat{E}\left(\ln f_{B}\right)=$ & 200 & 0.032 & 0.032 & 0.053 & 0.089 & 0.089 & 0.037 & 0.100 & 0.050 & 0.119 & 0.105 & 0.114 \\
-1.822 & 500 & 0.021 & 0.021 & 0.037 & 0.048 & 0.047 & 0.022 & 0.047 & 0.023 & 0.055 & 0.089 & 0.085 \\
& 1000 & 0.018 & 0.018 & 0.040 & 0.040 & 0.040 & 0.021 & 0.038 & 0.021 & 0.043 & 0.065 & 0.071 \\
$\hat{E}\left(\ln f_{C}\right)=$ & 200 & 0.299 & 0.296 & 0.247 & 0.394 & 0.392 & 0.361 & 0.357 & 0.345 & 0.391 & 0.325 & 0.410 \\
-3.072 & 500 & 0.121 & 0.121 & 0.129 & 0.226 & 0.226 & 0.220 & 0.223 & 0.197 & 0.263 & 0.230 & 0.327 \\
& 1000 & 0.084 & 0.084 & 0.101 & 0.161 & 0.161 & 0.140 & 0.144 & 0.135 & 0.187 & 0.163 & 0.255 \\
$\hat{E}\left(\ln f_{D}\right)=$ & 200 & 0.256 & 0.254 & 0.281 & 0.260 & 0.260 & 0.258 & 0.487 & 0.417 & 0.488 & 0.268 & 0.461 \\
-3.850 & 500 & 0.219 & 0.221 & 0.249 & 0.240 & 0.240 & 0.217 & 0.333 & 0.298 & 0.345 & 0.240 & 0.385 \\
& 1000 & 0.149 & 0.149 & 0.150 & 0.178 & 0.178 & 0.149 & 0.260 & 0.222 & 0.274 & 0.173 & 0.299 \\
\hline
\end{tabular}


Table 3: Numerical mean and standard deviation of ISEs for $f_{D}(\cdot)$. 'PI' refers to the plug-in method, and 'NRR' the normal reference rule. Values in parentheses are the corresponding standard deviations.

\begin{tabular}{|l|ccc|ccc|ccc|}
\hline $\begin{array}{l}\text { sample } \\
\text { size }\end{array}$ & \multicolumn{3}{|c|}{ mean } & \multicolumn{3}{|c|}{ standard deviation } & \multicolumn{3}{|c|}{ difference between ISEs } \\
& $\begin{array}{c}\text { MCMC } \\
(1)\end{array}$ & PI & NRR & MCMC & PI & NRR & $(1)-(2)$ & $(1)-(3)$ & $(2)-(3)$ \\
\hline 200 & 0.0077 & 0.0092 & 0.0176 & 0.00199 & 0.00136 & 0.00097 & -0.00152 & -0.00998 & -0.00847 \\
& & & & & & & $(0.00177)$ & $(0.00151)$ & $(0.00085)$ \\
500 & 0.0065 & 0.0060 & 0.0149 & 0.00179 & 0.00085 & 0.00061 & 0.00047 & -0.00842 & -0.00889 \\
& & & & & & & $(0.00155)$ & $(0.00147)$ & $(0.00058)$ \\
1000 & 0.0049 & 0.0041 & 0.0128 & 0.00123 & 0.00057 & 0.00045 & 0.00081 & -0.00789 & -0.00870 \\
& & & & & & & $(0.00107)$ & $(0.00099)$ & $(0.00032)$ \\
\hline
\end{tabular}

Table 4: $M C M C$ results for data generated from $f_{E}(\cdot)$ with sample size 1500.

\begin{tabular}{|l|c|r|c|c|r|l|}
\hline & bandwidths & mean & $\begin{array}{l}\text { standard } \\
\text { deviation }\end{array}$ & $\begin{array}{l}\text { batch-mean } \\
\text { standard error }\end{array}$ & \multicolumn{1}{|c|}{ SIF } & $\begin{array}{l}\text { acceptance } \\
\text { rate }\end{array}$ \\
\hline diagonal & $1 / b_{11}$ & 0.56 & 0.03 & 0.0009 & 21.85 & 0.250 \\
matrix & $1 / b_{22}$ & 0.58 & 0.03 & 0.0009 & 24.34 & \\
& $1 / b_{33}$ & 0.56 & 0.03 & 0.0009 & 29.25 & \\
& $1 / b_{44}$ & 0.58 & 0.03 & 0.0010 & 36.42 & \\
& $1 / b_{55}$ & 0.58 & 0.03 & 0.0009 & 34.14 & \\
\hline full & $b_{11}$ & 1.81 & 0.10 & 0.0042 & 41.83 & 0.272 \\
& $b_{21}$ & -0.15 & 0.15 & 0.0106 & 130.54 & \\
& $b_{22}$ & 1.73 & 0.09 & 0.0033 & 36.26 & \\
& $b_{31}$ & 0.11 & 0.18 & 0.0143 & 155.34 & \\
& $b_{32}$ & -0.15 & 0.13 & 0.0076 & 85.27 & \\
& $b_{33}$ & 1.80 & 0.10 & 0.0031 & 25.31 & \\
& $b_{41}$ & -0.12 & 0.14 & 0.0084 & 93.56 & \\
& $b_{42}$ & -0.09 & 0.14 & 0.0099 & 133.07 & \\
& $b_{43}$ & -0.02 & 0.14 & 0.0083 & 93.30 & \\
& $b_{44}$ & 1.74 & 0.10 & 0.0041 & 46.56 & \\
& $b_{51}$ & 0.00 & 0.14 & 0.0084 & 88.95 & \\
& $b_{52}$ & 0.07 & 0.14 & 0.0098 & 120.43 & \\
& $b_{53}$ & 0.05 & 0.16 & 0.0114 & 134.69 & \\
& $b_{54}$ & 0.18 & 0.13 & 0.0087 & 103.13 & \\
& $b_{55}$ & 1.78 & 0.10 & 0.0042 & 47.31 & \\
\hline
\end{tabular}


Table 5: Estimated Kullback-Leibler information for multivariate densities.

\begin{tabular}{|l|r|ccccccc|}
\hline & sample & \multicolumn{7}{|c|}{ Kullback-Leibler information } \\
& size & $M_{1}$ & $M_{2}$ & $M_{3}$ & $M_{4}$ & $M_{5}$ & $M_{6}$ & $N_{1}$ \\
\hline$\hat{E}\left(\ln f_{E}\right)=$ & 500 & 0.178 & 0.177 & 0.539 & 0.441 & 0.441 & 0.186 & 1.262 \\
-7.9283 & 1000 & 0.127 & 0.126 & 0.505 & 0.304 & 0.304 & 0.162 & 1.235 \\
\hline & 1500 & 0.118 & 0.117 & 0.470 & 0.276 & 0.276 & 0.141 & 1.545 \\
$\hat{E}\left(\ln f_{F}\right)=$ & 500 & 0.224 & 0.224 & 0.548 & 0.223 & 0.223 & 0.381 & 1.772 \\
-7.7934 & 1000 & 0.148 & 0.148 & 0.438 & 0.144 & 0.144 & 0.303 & 1.604 \\
\hline & 1500 & 0.152 & 0.151 & 0.402 & 0.149 & 0.149 & 0.291 & 1.571 \\
$\hat{E}\left(\ln f_{G}\right)=$ & 500 & 0.774 & 0.771 & 1.147 & 0.746 & 0.746 & 0.915 & 2.222 \\
-9.2232 & 1000 & 0.687 & 0.685 & 1.149 & 0.677 & 0.677 & 0.846 & 1.862 \\
\hline & 1500 & 0.696 & 0.696 & 1.029 & 0.679 & 0.680 & 0.845 & 1.992 \\
$\hat{E}\left(\ln f_{H}\right)=$ & 500 & 0.182 & 0.180 & 0.668 & 0.335 & 0.334 & 0.206 & 1.319 \\
-7.5123 & 1000 & 0.141 & 0.140 & 0.466 & 0.272 & 0.272 & 0.153 & 1.112 \\
\hline & 1500 & 0.127 & 0.126 & 0.423 & 0.242 & 0.242 & 0.148 & 1.100 \\
$\hat{E}\left(\ln f_{I}\right)=$ & 500 & 0.288 & 0.282 & 0.725 & 0.479 & 0.479 & 0.247 & 1.342 \\
-7.3760 & 1000 & 0.142 & 0.141 & 0.662 & 0.331 & 0.331 & 0.166 & 1.204 \\
& 1500 & 0.109 & 0.109 & 0.537 & 0.270 & 0.270 & 0.147 & 1.318 \\
\hline
\end{tabular}

Table 6: Numerical MISEs for the 5-dimension density $f_{H}(\cdot)$.

\begin{tabular}{|l|cc|cc|}
\hline \multirow{2}{*}{$\begin{array}{l}\text { sample } \\
\text { size }\end{array}$} & \multicolumn{2}{|c|}{ MISE } & \multicolumn{2}{c|}{ difference between ISEs } \\
\hline 500 & 0.000195 & 0.000499 & -0.000304 & 0.000023 \\
1000 & 0.000144 & 0.000421 & -0.000278 & 0.000015 \\
1500 & 0.000125 & 0.000391 & -0.000265 & 0.000008 \\
\hline
\end{tabular}


Table 7: $M C M C$ results obtained from the Earthquake data.

\begin{tabular}{|l|c|r|c|l|r|l|}
\hline & bandwidths & mean & $\begin{array}{l}\text { standard } \\
\text { deviation }\end{array}$ & $\begin{array}{l}\text { batch-mean } \\
\text { standard error }\end{array}$ & SIF & $\begin{array}{l}\text { acceptance } \\
\text { rate }\end{array}$ \\
\hline diagonal & $1 / b_{11}$ & 0.003 & 0.0001 & 0.000003 & 9.07 & 0.254 \\
matrix & $1 / b_{22}$ & 0.003 & 0.0001 & 0.000003 & 12.60 & \\
& $1 / b_{33}$ & 0.715 & 0.0383 & 0.000873 & 12.96 & \\
\hline full & $b_{11}$ & 311.65 & 0.07 & 0.002 & 15.80 & 0.246 \\
matrix & $b_{21}$ & 101.53 & 0.10 & 0.005 & 62.21 & \\
& $b_{22}$ & 388.57 & 0.10 & 0.003 & 15.84 & \\
& $b_{31}$ & 147.45 & 0.13 & 0.008 & 89.38 & \\
& $b_{32}$ & 97.21 & 0.16 & 0.011 & 118.86 & \\
& $b_{33}$ & 1.65 & 0.27 & 0.012 & 47.54 & \\
\hline
\end{tabular}

Table 8: CPU time for samplers of diagonal and full bandwidths (in minutes).

\begin{tabular}{|r|c|c|c|c|}
\hline sample & \multicolumn{2}{|c|}{ dimension=2 } & \multicolumn{2}{c|}{ dimension=5 } \\
\cline { 2 - 5 } size & diagonal matrix & full matrix & diagonal matrix & full matrix \\
\hline 200 & 2 & 2 & - & - \\
500 & 14 & 15 & 19 & 26 \\
1000 & 54 & 56 & 77 & 102 \\
1500 & - & - & 177 & 238 \\
\hline
\end{tabular}


Figure 1: Contour graphs of the proposed bivariate densities.

Density A

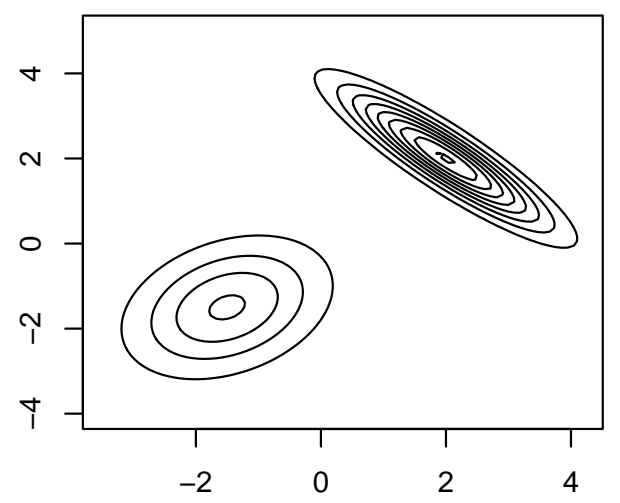

Density C

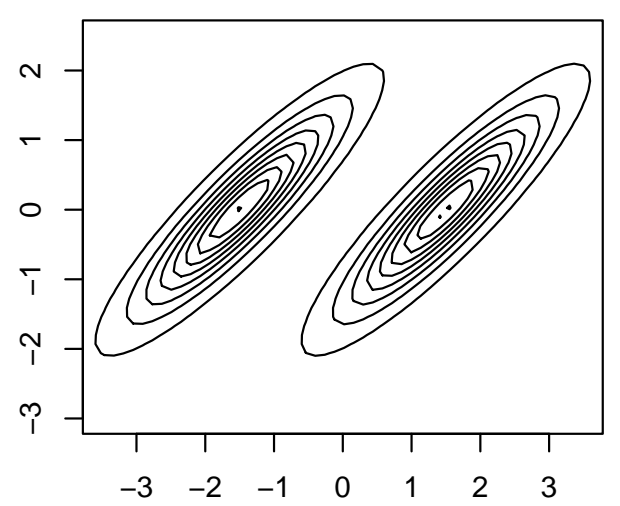

Density B

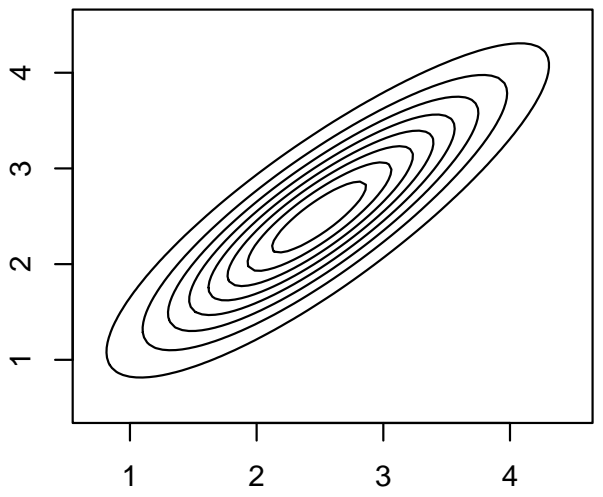

Density D

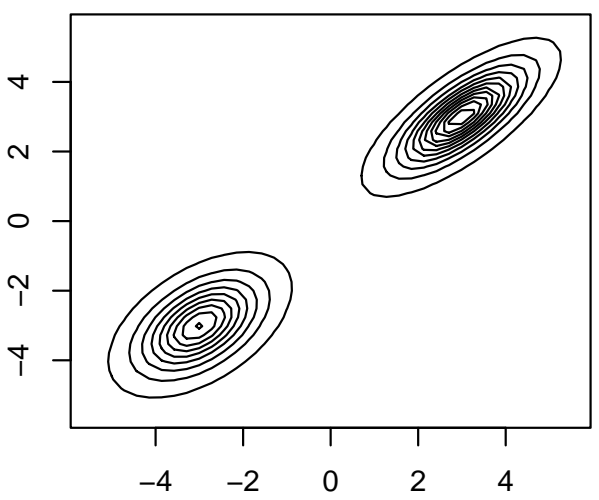


Figure 2: The 98\% highest density region for the earthquake data showing four views looking from north, east, south and west. Negative log-depth is on the vertical axis, and various combinations of latitude and longitude are on the horizontal axes.
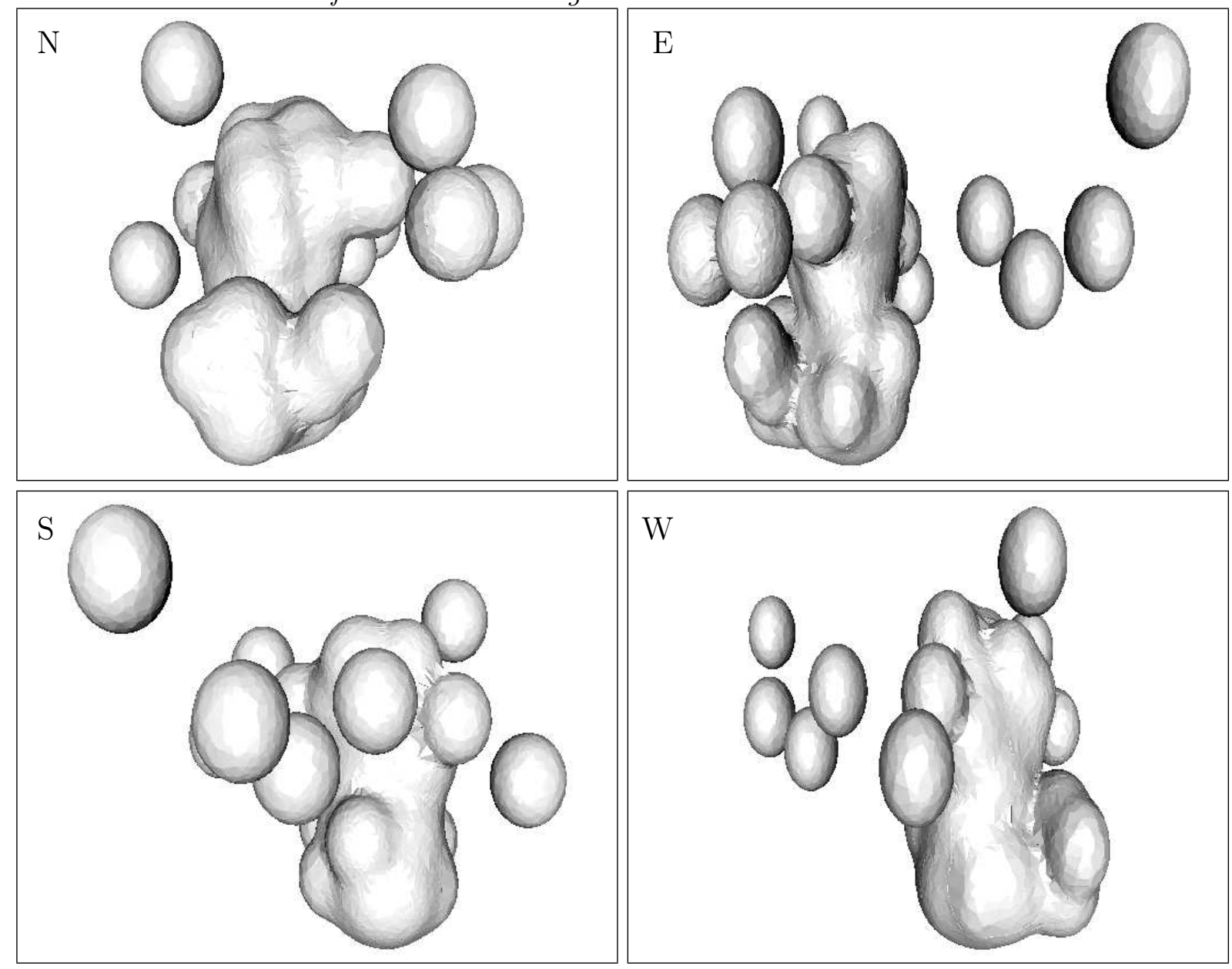